http://jmscr.igmpublication.org/home/ ISSN (e)-2347-176x ISSN (p) 2455-0450 crossref DOI: https://dx.doi.org/10.18535/jmscr/v8i2.06

\author{
Deurnal Of Medical Science And Clinical Research

\title{
An Atypical Presentation of ODS - Case Report
}

\author{
Authors \\ Aida Mary Varghese ${ }^{1}$, Muhammed Rafath $\mathbf{E}^{2}$, Parvathy $\mathbf{R S}^{3}$, \\ Neethu Varghese ${ }^{4}$, Suresh Raghavan ${ }^{5}$ \\ 1,2,3,4 Junior Resident, Department of General Medicine, Govt. T. D. Medical College, Alappuzha, Kerala, \\ India \\ ${ }^{5}$ Professor, Department of General Medicine, Govt. T. D. Medical College, Alappuzha, Kerala, India
}

\begin{abstract}
Osmotic demyelination syndrome is a disorder characterized by wide spread development of demyelination in the pontine as well as extra pontine regions. It has been a recognized complication of rapid correction of hyponatremia. However, a variety of other conditions have been associated with the development of ODS, independent of changes in serum sodium. Here we present a patient who came with features suggestive of $O D S$, uncontrolled hyperglycemia and recently corrected hyponatremia.
\end{abstract}

\section{Introduction}

Osmotic Demyelination Syndrome (ODS) is a life threatening demyelinating syndrome, which usually occurs in the setting of a rapid correction of severe hyponatremia ${ }^{(1)}$. The key factor in ODS pathogenesis is a rapid change in serum osmoles. Despite the pronounced fluctuations in serum osmolality, ODS is rarely seen in diabetics. Patient may develop spastic quadriplegia, pseudo bulbar palsy and varying degree of encephalopathy or coma.

The pathology consists of demyelination without inflammation in the base of pons, with relative sparing of axons and nerve cells.

The pathogenesis of ODS involves the inability of brain cells to respond to rapid changes in osmolality of interstitial compartment of brain, leading to dehydration of energy dependent cells with subsequent axonal damage.

MRI is the imaging technique of choice for diagnosing ODS.

\section{Case Report}

A 50 year old female patient, known case of systemic hypertension and type 2 diabetes mellitus on irregular treatment presented with burning abdominal pain and recurrent episodes of vomiting for 4 days followed by features of metabolic encephalopathy in the form of altered sensorium without fever, seizures or focal neurological deficit

$\mathrm{O} / \mathrm{E}$ patient was disoriented, vitals were normal, cranial nerves including fundus examination was normal, motor system examination was normal, other system examination was normal. We clinically suspected the possibility of metabolic encephalopathy and was investigated.

Investigations revealed an RBS of 300, serum electrolytes $(\mathrm{Na} / \mathrm{K})$ were 106 and $3.8, \mathrm{HbA} 1 \mathrm{c}$ 9,RFT 41/1.4. Other investigations including CT Brain was normal. She was diagnosed as acute gastritis with severe hyponatremia with metabolic 
encephalopathy non convulsive with no features of raised intracranial pressure

Patient was treated with hypertonic saline as per the standard guidelines and other symptomatic measures including insulin, iv fluids and other supportive measures

Sodium correction was given at a rate not exceeding $8 \mathrm{mmol} /$ day and serum sodium of 135 was achieved over a period of 7 days. Patient improved and was discharged with complete recovery, in a totally asymptomatic state.

By 3 rd day of her discharge she developed generalised slowing of activities, slowing of gait, along with paucity of upper and lowerlimbs and hypophonia which were suggestive of acute parkinsonian features.

On examination, patient was fully conscious, vitals were stable, higher mental function examination showed hypophonic dysarthria with difficulty in swallowing, depressed mood, emotional incontinence, vacant stare with infrequent blinking, expressionless face

Motorsystem examination showed bilateral hypertonia suggestive of lead pipe rigidity, deep tendon reflexes-brisk bilaterally with no clonus, plantar reflex- extensor bilaterally, grade 4 power both upper limbs and lower limbs.

Gait - slow shuffling gait with reduced arm swing and some ataxic component.Oro facial involuntary movements were present.

Fundus - normal. There was no neck stiffness. Skull and spine were normal.

Examination of other systems was normal

Clinically patient had an acute presentation after treatment of metabolic encephalopathy with complete recovery and had features ofbilateral pyramidal signs, bilateral cerebellar signs, bilateral extra pyramidal signs, emotional incontinence

Clinically we suspected the possibility of osmotic demyelination syndrome because of the following reasons:

1. Chronic hyperosmolar state (uncontrolled diabetes)
2. Recent treated case of hyponatremic encephalopathy

3. Sudden onset of bilaterally symmetrical neurological signs.

Investigations- BRE, LFT -normal, Urea- 42, creatinine -1.4 ,

URE- sugar -1.5, acetone- negative, RBS - 360, SE- 135/4.8,T.Protein/Albumin- 7/3.9, HbA1c -9, ANA profile- negative, APLA -negative, pANCA, cANCA -negative,

CRP- negative, ESR -20, peripheral smearnormal, serum electrophoresis- normal,

Chest Xray was normal, ECG was normal, CSF study was normal

MRI BRAIN showed FLAIR/ T2 hyperintensity with diffusion hyperintensity in the central pons, bilateral caudate and lentiform nucleus and lateral thalamus suggestive of osmotic demyelination syndrome

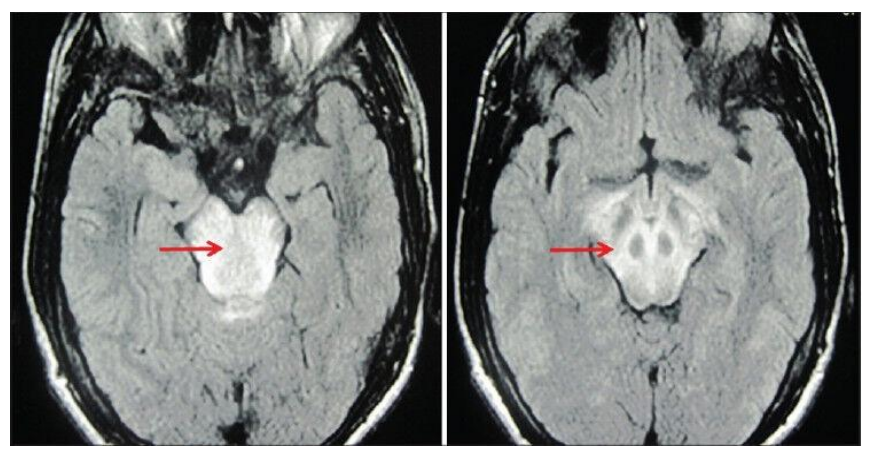

Magnetic resonance imaging of the brain at onset showing hyperintensities (red arrows) in T2WI in the areas of brainstem suggestive of osmotic demyelination

Diagnosis, outcome and follow up

After MRI confirmation the patient was diagnosed with osmotic demyelination syndrome. In this case the most likely mechanism of ODS could be subacute changes in brain secondary to hyperosmolar hyperglycemia resulting in osmotic demyelination

Patient was managed with insulin, methyl cobalamine, antiparkinsonian drugs, steroids and other supportive measures and patient symptomatically improved 


\section{Discussion}

ODS is a disorder characterized by the widespread development of demyelination in the pontine as well as the extra pontine regions.

Demyelination (non inflammatory) of the central pons (CPM) is one manifestation of ODS. (EPM) extrapontine myelinolysis seen in $10 \%$ cases mostly involves basal ganglia and thalamus ${ }^{(2)}$

ODS usually occurs with rapid correction of hyponatremia (rise in sodium level by > $12 \mathrm{mmol} /$ day). Alcoholism, malnourishment and severe debilitating illnesses are risk factors for the development of ODS

Common sites of osmotic demyelination are Pons, Cerebellum and Basal ganglia with the maximum grey and white matter elements making it more susceptible

\section{Mechanism}

In chronic hyponatremia brain cells lose electrolytes and osmotically active organic osmolytes (such as myoinositol, glutamate and glutamine) over a few days rendering the cells isotonic to the extracellular fluid to maintain cell volume and provide protection against brain cell swelling by a process called cerebral adaptation. ${ }^{(3)}$ During rapid correction of hyponatremia brain cells, especially oligodendrocytes shrink due to volume loss as they cannot synthesize or transport osmoles at such rapid rates leading to osmotic demyelination. ${ }^{(4)}$ Osmotic shrinkage of endothelial cells opens the blood brain barrier allowing the entry of complement and other cytotoxic components .These changes may directly injure and induce apoptosis of astrocytes, leading to a disruption in the function of myelin producing oligodendrocytes, release of inflammatory cytokines and activation of microglia

\section{Risk Factors include}

Rapid correction of severe hyponatremia, alcoholism, c/c liver disease, malnutrition, uremia, hyperglycemia, hypokalemia
Pathogenesis of ODS in Hyerosmolar

\section{Hyperglycemia}

Theories proposed behind osmotic demyelination in the setting of hyperosmolar hyperglycemia are: ${ }^{(5)}$

1) Subacute changes in brain cells secondary to hyperglycemia leading to demyelination.

2) Rapid changes in osmolality associated with the fluctuations in serum glucose causing osmotic demyelination.

3) Hypertonic insult associated with hyperglycemia itself causing demyelination.

\section{Clinical Presentation}

A biphasic clinical course is seen in ODS, initially encephalopathic phase followed by deterioration later. The clinical manifestation vary based on area of brain involvement and include dysarthria and dysphagia with corticobulbar fibre involvement, flaccid quadriparesis with corticospinal tract involvement and in severe cases locked in syndrome. Tremor, ataxia, movement disorders, psychiatric and behavioral change, mutism, parkinsonism, dystonia, catatonia may be seen with extrapontine involvement. ${ }^{(6)}$

\section{Prevention and Treatment}

Gradual correction of hyponatremia is the most important step in the management of hyponatremic patients

Among patients with hyponatremia lasting more than 2-3 days or of unknown duration serum $\mathrm{Na}$ should be raised by less than 6-8 meq/l in any 24 hr period to prevent ODS.

Treatment of ODS is mainly supportive. Relowering the serum sodium may be beneficial if initiated early in ODS caused by rapid correction of severe chronic hyponatremia. Other recommendations include steroids, minocycline, intravenous immunoglobulin. There is no specific treatment other than supportive management for non sodium dependendent osmotic demyelination. 


\section{Conclusion}

Though our patient received standard correction of symptomatic hyponatremia she developed ODS, probably due to already existing uncontrolled hyperglycemia.

This case report of ODS associated with hyperglycemia highlights the importance of adequate blood glucose control in diabetics.

We should be aware of complications like ODS in diabetics which can present atypically and is only diagnosed in the presence of a high index of clinical suspicion

\section{Bibliography}

1. Adams RD, Victor M, Mancall EL. Central Pontine Myelinolysis: A Hitherto Undescribed Disease Occurring in Alcoholic and Malnourished Patients. AMA Arch Neurol Psychiatry. 1959 Feb 1;81(2):154-72.

2. Osmotic demyelination syndrome due to hyperosmolar hyperglycemia [Internet]. [cited 2020 Jan 22]. Available from: https://www.mdedge.com/ccjm/article/168 893/neurology/osmotic-demyelinationsyndrome-due-hyperosmolarhyperglycemia

3. Martin RJ. Central pontine and extrapontinemyelinolysis: the osmotic demyelination syndromes. $\mathbf{J}$ Neurol Neurosurg Psychiatry. 2004 Sep 1;75(suppl 3):iii22-8.

4. DeLuca G, Nagy Z, Esiri M, Davey P. Evidence for a role for apoptosis in central pontinemyelinolysis. ActaNeuropathol (Berl). 2002 Jun 1;103(6):590-8.

5. Talluri S, Charumathi R, Khan M, Kissell $\mathrm{K}$. Atypical presentation of central pontinemyelinolysis in hyperglycemia. Endocrinol Diabetes Metab Case Rep [Internet]. 2017 Sep 4 [cited 2020 Jan 22];2017. Available from: https://www.ncbi.nlm.nih.gov/pmc/articles /PMC5592705/
6. Sullivan AA, Chervin RD, Albin RL. Parkinsonism after correction of hyponatremia with radiological central pontinemyelinolysis and changes in the basal ganglia.J ClinNeurosci. 2000 May 1;7(3):256-9. 